\title{
Persian and Malay: 1000 Words in Common
}

\author{
Abolfazl Shirban Sasi \\ Department of Applied Foreign Languages \\ TransWorld University \\ Taiwan
}

\begin{abstract}
Today, varieties of the Malay language are spoken by more than two hundred million people, mostly in Indonesia and Malaysia. Also, more than one hundred million people speak Persian dialects in Iran, Afghanistan, and Tajikistan. Throughout centuries, Malay has been influenced by other languages such as Arabic, Dutch, Portuguese, and English. Likewise, Persian has borrowed many vocabularies from Arabic and European languages. Since words are one of the most important elements in mastering any language, a concise classification of the common Persian and Malay words has vast pedagogical implications. In this study, more than 1000 Malay words that were recited to 64 Iranian university students on various occasions were recognized by them to be almost completely homophonic to Persian words. Most of these common terms were then classified into five main categories the biggest of which is those words which have exactly the same meaning, and almost identical pronunciation.
\end{abstract}

Keywords: Arabic; Homophonic; Farsi; Malay; Persian

\section{Introduction}

This study was carried out during the researcher's stay in Malaysia when doing his $\mathrm{Ph}$. D. degree. Being an Iranian, he came across several instances of Persian words which were almost identically used by the Malay. Below are the main sources which finally led to the current study:

- Mass media such as TV/radio programs, newspapers, etc.

- Interactions with Malay native speakers

- Malay books and dictionaries

- Educational lectures delivered in Malay

- English movies with Malay subtitles

- Various signage used in public places like banks, hospitals, streets, universities, airports, malls, etc.

- Malay catalogues and brochures

More than two hundred million people in Indonesia, Malaysia, southern Thailand, Singapore, and Brunei speak dialects of the Malay language. Also, more than one hundred million people speak Persian varieties in Iran, Afghanistan, and Tajikistan (The World Factbook a \& b; Katzner, 2002). It is very interesting that we can observe so many Farsi (Persian) words that are somehow used in the Malay, given the fact that these languages belong to two very different language families, i.e. Austronesian, and Indo-European. In this study, the researcher does not intend to sort out the etymology of the words, rather their being used by Persian speakers is the focus of this paper. Thus, whatever the root of the terms, the criterion in this research is that the word should be comprehensible when heard by Persian native speakers. Excluding a lot of technical terms, jargons and proper nouns which are almost equally used in both languages, the researcher has classified hundreds of shared vocabulary into word groups with detailed properties.

As mentioned, this study does not focus on the etymology of the words in question; however, since Islam is the dominant religion in all the countries where Malay and Persian are spoken, and also because Arabic is the language of Islam, the researcher postulates that most of the common words in Malay and Persian have Arabic roots. Since the dawn of Islam in the Arabian Peninsula in the $7^{\text {th }}$ century A.D., Arabic, a Semitic language, has effected (or in some cases, replaced) numerous languages in Asia and North Africa (Kartzner, 2002). Likewise, both Malay and Persian have borrowed many common words from Arabic. This might be the most influential cause for the existence of the common vocabulary in these two languages. 
Persian has also adopted the Arabic script (alphabet). Thus, both languages are written from right to left. However, there are two big differences between Arabic and Persian: 1) In Arabic we use diacritics; namely, marks

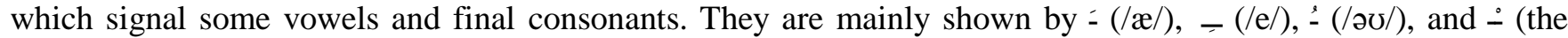
consonant is not followed by a vowel). Persian, on the other hand, does not use these symbols. Thus, Persian spelling and pronunciation are more difficult to learn. 2) Persian alphabet contains four more letters/consonant sounds. They are: $\longleftarrow / \mathrm{p} /, j / 3 /, \xi / \mathrm{g} /$, and $\mathrm{j} / \mathrm{t} /$.

Malay also used to be written in Arabic alphabets called Jawi which was introduced to the region by Muslim missionaries in the $15^{\text {th }}$ century (Sulaiman, 2005). In the $19^{\text {th }}$ century, the British developed a Roman-based alphabet which is in general use now. Affixes as we use them are somehow absent in Malay. That is, Malay grammatical functions are accomplished by adding an extra word (Byrnes \& Suan, 2006; Katzner, 2002).

\section{Method}

The researcher first scrutinized all the Malay-English section of a 45,000-entry dictionary (Hawkins, 2006) to come up with a primary list of words in Malay which sounded like Persian words. Some were exactly identical and also frequently used equally in both languages. Yet, some other words were pronounced with a little or some resemblance. Interestingly, there are also some high frequent Malay words which sound exactly like Persian vulgar/taboo terms. These words occasionally cause embarrassing situations when Iranians hear them. The English equivalents of a few of these Malay words are: cost, empty, former, key, left, rat, sea bird, and zero. The researcher did not include such words in the word list.

Consequently, 1300 words were filtered at the first stage. Then before cross-examining the words, the researcher randomly added some 200 more Malay words to come up with a 1500-item word list. This final word addition was to create distraction from the other Malay-Persian homophonic words on the list. Then the Author randomly put these words in 25 sets, each containing 60 words.

The next step was administering the 25 sets of words to Iranian university students mostly in University Sains Malaysia (USM). Three Malay graduate students (two women and one man) helped the researcher to conduct this phase of the study. On different occasions, the Iranian students were requested to fill out a form. The form was a numbered blank list of 60 words. The students were asked to listen carefully to the Malay person who would read them the Malay words in a natural tone twice. The instructions on the answer sheet requested them to write down the Persian word for each item whenever they could recognize it from the Malay. If not applicable, the students were simply asked to leave that item unmarked. The research participants were kept anonymous.

Each set of words were administered 10 times. Totally, 64 Iranian students (41 women and 23 men) were consulted in this study. The whole administration procedure took about five months. The recitationsessions took place in fairly quite places like researcher's apartment, classrooms, and even restaurants! Precautions were taken so that one certain individual would not be administered the same set of words twice. The maximum number of participants in each session was eight students.

\section{Results}

Totally, 1,073 Malay words were recognized by the Iranian participants in this study as having either identical or very similar pronunciation to Persian words. Each of these words was perceived and written down on the forms 7 to 10 times. There was no particular reason statistically in choosing this number. This merely means that the above-mentioned words were recognized as their Persian homophonic counterparts at least for 70 percent of the time. Subsequently, the words which were recognized less than seven times were not considered qualified for further analysis. In the next step, the Author tried to cross out all technical terms, jargons, as well as proper nouns of people and places. Finally, 751 Malay-Persian homophones were classified into five major categories (APPENDICES A, B, C, D, E). The word lists are presented in the appendices because they were very long and could not be presented in the main body of the paper. For each entry in these lists, a Persian phonemic/broad transcription (Richards, Platt, \& Platt, 1992) is given. Also, in case of the compound Malay words or expressions, the Farsi-sounding segment (the Keyword) is shown in bold letters. Table 1 summarizes these five categories with their characteristics, the word count, as well as the ratio of each type of words to the whole sum in this paper. 
Table 1: Malay-Persian homophonic words in this paper

\begin{tabular}{|c|c|c|c|}
\hline Group & Word Characteristics & $\begin{array}{l}\text { Word } \\
\text { Count }\end{array}$ & $\begin{array}{l}\text { Word } \\
\text { Count } \\
\text { Ratio (\%) }\end{array}$ \\
\hline 1 & $\begin{array}{l}\text { - identical or very similar pronunciation; } \\
\text { - exactly the same meaning; } \\
\text { - the same part of speech. }\end{array}$ & 519 & 69 \\
\hline 2 & $\begin{array}{l}\text { - identical or very similar pronunciation; } \\
\text { - exactly the same meaning; } \\
\text { - different part of speech. }\end{array}$ & 37 & 5 \\
\hline 3 & $\begin{array}{l}\text { - compound Malay words, or expressions a } \\
\text { segment of which sounds identical or very } \\
\text { similar to Persian; } \\
\text { - the segment carries exactly the same or very } \\
\text { similar meaning to the Persian Keyword. }\end{array}$ & 114 & 15 \\
\hline 4 & $\begin{array}{l}\text { - identical or very similar pronunciation; } \\
\text { - somehow related shades of meaning; } \\
\text { - part of speech might vary. }\end{array}$ & 38 & 5 \\
\hline \multirow[t]{2}{*}{5} & $\begin{array}{l}\text { - identical or very similar pronunciation; } \\
\text { - different meanings; } \\
\text { - part of speech not applicable. }\end{array}$ & 43 & 6 \\
\hline & SUM & 751 & 100 \\
\hline
\end{tabular}

\section{Conclusion and Recommendations for Further Study}

This study has focused on the identical or similar vocabulary used in Malay and Persian. The results reavealed that even two apparently distant languages by their origions can share many similarities, or at least common lexicon. The findings of this research can help speakers of both languages enjoy learning the counterpart toungue at higher levels and with more comfort. The Author also recommends the following topics for future undertakings:

- Contrastive studies between Malay and Persian concerning syntax, semantics, or pragmatics;

- Contrastive study between the Malay and Persian borrowed words;

- Contrastive study between Malay and Persian with reference to the etymology of the common words;

- Contrastive study on the impact of the official religions practiced in Iran and Malaysia on the usage of the common vocabulary;

- IPA transcription of the Malay words in the published dictionaries.

\section{References}

Byrnes, C., \& Suan, T. L. (2006). Teach yourself Malay. Oxon: The McGraw-Hill Companies, Inc.

Hawkins, J. M. (ed.). (2006). Kamus Dwibahasa Oxford Fajar Inggeris-Melayu/Melayu- Inggeris. Selangor: Oxford Fajar Sdn. Bhd.

Katzner, K. (2002). The languages of the world. London: Routledge and Kegan Paul Ltd.

Richards, J. C., Platt, J., \& Platt, H. (1992). Longman dictionary of language teaching and applied linguistics. Essex: Longman Group UK Limited.

Sulaiman, O. (2005). Malay for everyone. Selangor: Pelanduk Publications.

THE WORLD FACTBOOK (a). Field listing: Languages. Retrieved from

https://www.cia.gov/library/publications/the-world-factbook/fields/2098.html (Retrieved in October, 2017)

THE WORLD FACTBOOK (b). Country Comparison: Population. Retrieved from

https://www.cia.gov/library/Publications/the-world-factbook/rankorder/2119rank.html (Retrieved in October, 2017) 


\section{APPENDIX A: Word Group 1}

Identical or very similar pronunciation, same meaning, and the same part of speech

\begin{tabular}{|c|c|c|c|}
\hline Malay Word & $\begin{array}{l}\text { English } \\
\text { Equivalent }\end{array}$ & $\begin{array}{l}\text { Persian } \\
\text { Pronunciation }\end{array}$ & $\begin{array}{l}\text { Persian } \\
\text { Key Word }\end{array}$ \\
\hline abadi (adj) & eternal & /æbæ'di:/ & ابدى \\
\hline abid (n) & devout person & /æ'bi:d/ & عبيد \\
\hline abjad (n) & alphabet & /æb'dzæd/ & ابجد \\
\hline abses (n) & swelling & /pb'se/ & آبسه \\
\hline $\operatorname{adas}(\mathrm{n})$ & lentils & /æ'dæs/ & عدس ع ع \\
\hline adat (n) & convention & /p'dæt/ & 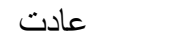 \\
\hline adil (adj) & fair & /v'del/ & عادل عال \\
\hline afiat (adj) & healthy & /p' fiæt/ & عافيت \\
\hline ahli (n) & member & /æhl/ & اهل \\
\hline aib (n) & flaw & /erb/ & عيب \\
\hline aid (n) & feast & /eId/ & عيد \\
\hline ajaib (adj) & strange & /æ'dzi:b/ & عجيب \\
\hline ajal (n) & death & /æ'dzæl/ & اجل \\
\hline akal (n) & ability to think & /æql/ & عقل \\
\hline akbar (adj) & almighty & /æk'bær/ & اكبر \\
\hline akhir (adj) & final & /æ'xi:r/ & اخير \\
\hline akhirat (n) & the next world & /pxe'ræt/ & آخرت \\
\hline akibat (n) & consequence & /pqe'bæt/ & عاقبت \\
\hline akidah (n) & belief & /æqi'de/ & عقيده \\
\hline akil (adj) & intelligent & /p'qel/ & عاقل ع \\
\hline $\operatorname{alam}(\mathrm{n})$ & world & /p'læm/ & عالم \\
\hline alamat (n) & sign & /ælp'mæt/ & علامت \\
\hline album (n) & album & /pl'bəom/ & آلبوم \\
\hline alim (adj) & pious & /p'lem/ & عالم \\
\hline alkimia (n) & alchemy & /ki'mip/ & كيميا \\
\hline alcohol (n) & alcohol & /æl'kəðl/ & الكل \\
\hline Allah (n) & God & /æl'lph/ & الله \\
\hline almarhum (n) & deceased man & /mær'hu:m/ & مرحوم \\
\hline $\operatorname{am}(\operatorname{adj})$ & general & /Ppm/ & عام \\
\hline amal (n) & action & /æ'mæl/ & عمل \\
\hline amali (adj) & practical & /æmæ'li:/ & عملى \\
\hline aman (adj) & peaceful & /æ'mon/ & 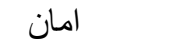 \\
\hline amanah (n) & property entrusted & /æmb'næt/ & امانت \\
\hline $\operatorname{amir}(\mathrm{n})$ & ruler & /æ'mi:r/ & امير اير \\
\hline ampul (n) & syringe & /om'pu:1/ & آميول \\
\hline $\operatorname{anasir}(\mathrm{n})$ & elements & /ænp'ser/ & عناصر \\
\hline anbia (n) & the prophets & /æn'bip/ & انبيا \\
\hline $\operatorname{anggur}(\mathrm{n})$ & grape & /æท'gu:r/ & انكور \\
\hline antena (n) & aerial & /pn'ten/ & آنتن \\
\hline antic (adj) & antique & /on'ti:k/ & آنتيك \\
\hline $\operatorname{arak}(\mathrm{n})$ & vodka & /æ'ræq/ & عرق \\
\hline arif (adj) & wise & /p'ref/ & عارف \\
\hline $\operatorname{artis}(\mathrm{n})$ & artist & /pr'ti:st/ & آرتيست \\
\hline Arya (adj) & Aryan & /prjp'?i:/ & آريايى \\
\hline $\operatorname{asal}(\mathrm{n})$ & origin & /æs1/ & اصل \\
\hline $\operatorname{asar}(\mathrm{n})$ & afternoon & /æsr/ & عصر \\
\hline asas (n) & foundation & /æ'sps/ & اساس \\
\hline asasi (adj) & fundamental & /æsp'si:/ & اساسى \\
\hline
\end{tabular}




\begin{tabular}{|c|c|c|c|}
\hline asphalt (n) & asphalt & /ps'fplt/ & آسفالت \\
\hline $\operatorname{askar}(\mathrm{n})$ & soldier & /æs'gær/ & عسخر \\
\hline asli (adj) & indigenous & /æ'sli:/ & اصلى \\
\hline aulia (n) & holy man & 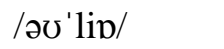 & اوليا \\
\hline aurat (n) & pubic areas & /əひ'ræt/ & اورت \\
\hline awal (adj) & beginning & /æv'væl/ & اول \\
\hline awam (adj) & public & /æ'vpm/ & عو ام \\
\hline ayat (n) & statement & $/ \mathrm{p}^{\prime} \mathrm{je} /$ & آيه \\
\hline azab (n) & punishment & /æ'zDpb/ & عذاب \\
\hline azali (adj) & eternal & /æzæ'li:/ & ازلى \\
\hline $\operatorname{azan}(n)$ & call to prayer & /æ'zDn/ & اذان \\
\hline bab (n) & chapter & /bpb/ & باب \\
\hline badam (n) & almond & /bp'dpm/ & بادام \\
\hline badan (n) & body & /bæ'dæn/ & بلن \\
\hline bahas (n) & debate & /bæhs/ & بحث \\
\hline bait (n) & couplet & /beit/ & بيت \\
\hline bakhil (adj) & stingy & /bæ'xi:1/ & بخيل \\
\hline balkoni (n) & balcony & /bol'kəon/ & بالكن \\
\hline bank (n) & bank & /bonk/ & بانك \\
\hline bapa (n) & father & /bp'bp/ & بابا با \\
\hline barzakh (n) & purgatory & /bær'zæx/ & برزخ \\
\hline batal (adj) & invalid & /bo'tel/ & باطل \\
\hline bateri (n) & battery & /bp'tri/ & باترى \\
\hline batin (n) & soul & /bo'ten/ & باطن \\
\hline bau (n) & smell & /bu:/ & بو \\
\hline bazaar (n) & market & /bp'zpDr/ & باز ار \\
\hline berkat (n) & blessing & /bær'kæt/ & بركت \\
\hline biadab (adj) & impolite & /bi: æ'dæb/ & بى ادب \\
\hline bibi (n) & aunt & /bi'bi/ & بى بى \\
\hline bidaah (n) & heresy & /be'dPæt/ & بدعت \\
\hline bilion (n) & billion & /bi'liu:n/ & بيليون \\
\hline $\operatorname{bin}(n)$ & son of & /ebn/ & ابن ابن \\
\hline binti (n) & daughter of & /bent/ & بنت \\
\hline bismillah (n) & in the name of & a /besmel'lah/ & بسم الله \\
\hline bom (n) & bomb & /bəumb/ & بمب \\
\hline brahman (n) & Hindu & /beræh'mæn/ & بر همن \\
\hline brother (n) & brother & /bæro'dær/ & بر ادر \\
\hline cadir (n) & thick soft quilt & $/ \mathrm{t} \int \mathrm{p}^{\prime}$ dəvr/ & جادر \\
\hline camca (n) & spoon & $/ \mathrm{t} \int æ m ' t \int e /$ & 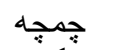 \\
\hline cangkuk (n) & hook & 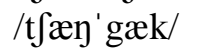 & جنغ \\
\hline $\operatorname{cek}(\mathrm{n})$ & check & $/ \mathrm{t} \int \mathrm{ek} /$ & "جى \\
\hline dajal (n) & evil & /dæ'dzæl/ & دجل \\
\hline dakwah (n) & preaching & /dæ?'væt/ & دعوت \\
\hline dalal (n) & agent & /dæl'lpl/ & د دلال \\
\hline dalil (n) & evidence & /dæ'li:1/ & دليل \\
\hline darjah(n) & degree & /dæræ'dze/ & درجه \\
\hline darjat (n) & status & /dæræ'dze/ & درجه \\
\hline darurat (n) & emergency & /zæru: 'ræt / & ضرورت \\
\hline darwis (n) & dervish & /dær'v i:f/ & درويش \\
\hline daulat (n) & sovereignty & /dəひ'læt/ & دولت \\
\hline deklamasi (n) & reading poems & /deklæ'me/ & دكلمه \\
\hline destar (n) & headdress & /dæs'tpr/ & دستار \\
\hline dewan (n) & hall & /di'vpn/ & ديوان \\
\hline
\end{tabular}




\begin{tabular}{|c|c|c|c|}
\hline diplomat (n) & diplomat & /dipləひ' mpt/ & دييلمات \\
\hline doa $(n)$ & payer & /dəひ'?p/ & " دعا \\
\hline doktor (n) & doctor & /dəuk'təur/ & 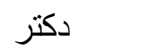 \\
\hline dunia (n) & world & /dəon'jp/ & دنيا \\
\hline duniawi (adj) & worldly & /dəonjæ'vi/ & دنيوى \\
\hline eau-de-cologne (n) & eau-de-cologne & /əudəukəu'ləun/ & ادوكلن \\
\hline eh (int.) & eh & /eh/ & ol \\
\hline ehwal (n) & matters & /æh'vpl/ & احوال احو \\
\hline ekspres (adj) & express & /ekspe'res/ & اكسيرس \\
\hline elemen (n) & element & /ele'mon/ & المان \\
\hline e-mel (n) & e-mail & /i:'merl/ & ايميل \\
\hline eskot (n) & escort & /es'kəurt/ & اسكورت \\
\hline faedah $(\mathrm{n})$ & benefit & /fpje'de/ & فايده \\
\hline faham (n) & understanding & /fæhm/ & فهم \\
\hline faharasat (n) & list & /feh'rest/ & فهرست \\
\hline fail (n) & file & /fail/ & فايل \\
\hline fajar (n) & dawn & /fædzr/ & فجر \\
\hline fakir (n) & poor person & /fæ' qi:r/ & فقير \\
\hline falak (n) & celestial sphere & /fæ'læk/ & فلى \\
\hline falsafah (n) & philosophy & /fælsæ'fe/ & فلسفه \\
\hline famili (n) & family & /fp'mi:1/ & فاميل \\
\hline Farsi (n) & Farsi & /for'si:/ & فارسى \\
\hline fasal (n) & clause & /fæsl/ & فصل \\
\hline fasih (adj) & fluent & /fæ'si:h/ & فصيح \\
\hline fasik (n) & sinner & /fo'seq/ & فاسق \\
\hline Fatihah (n) & Koran first chapter & /fpte'he/ & فاتحه \\
\hline fatwa (n) & Islamic law & /fæt'vp/ & فتوى \\
\hline fiil (n) & behavior & /fe?1/ & فعل \\
\hline fikir (n) & thinking & /fekr/ & فكر \\
\hline filem (n) & film & /film/ & فيلم \\
\hline firasah (n) & intuition & /ferp'sæt/ & فر است \\
\hline Firaun (n) & Pharaoh & /fer' Pəon/ & فر عون \\
\hline firdaus (n) & paradise & /fer'dəus/ & فردوس \\
\hline fitnah (n) & slander & /fet'ne/ & فتنه \\
\hline fitrah (n) & natural tendency & /fet'ræt/ & فطرت \\
\hline fizik (n) & physics & /fi'zi:k/ & فيزيك \\
\hline flut (n) & flute & /fu:'lu:t/ & فلوت \\
\hline fosil (n) & fossil & /fəひ'si:1/ & فسيل \\
\hline foto $(n)$ & photo & /fəひ'təo/ & فتو \\
\hline fotokopi (n) & photocopy & / fəðtəðkəひ'pi/ & فتو كيى \\
\hline gandum (n) & wheat & /gæn'dəom/ & كندم \\
\hline garaj $(n)$ & garage & /gn'roz/ & 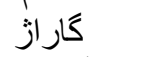 \\
\hline gari (n) & handcuff & /gn'ri:/ & كارى \\
\hline gas (n) & gas & /gpz/ & كاز \\
\hline genus (n) & gender & /dzens/ & جنس \\
\hline gerenti (n) & guarantee & /gpron'ti:/ & كار انتى \\
\hline ghaib (adj) & invisible & /qeib/ & غيب \\
\hline ghalib (adj) & normal & /qp'leb/ & غالب \\
\hline ghazal (n) & a musical genre & /qæ'zæl/ & غزل \\
\hline gimnastik (n) & gymnastic & /zimnos'ti:k/ & زُيمناستيك \\
\hline grafik (n) & graphic & /gern'fi:k/ & كر افيكى \\
\hline guni (n) & sack & /gu:'ni:/ & كَّنى \\
\hline had (n) & limit & /hæd/ & حد \\
\hline
\end{tabular}




\begin{tabular}{|c|c|c|c|}
\hline hadiah (n) & present & /hæ'diə/ & هدياه \\
\hline hadis (n) & Islamic traditions & /hæ'di:s/ & حديث \\
\hline haiwan (n) & animal & /hei'vpn/ & حيوان \\
\hline hajat (n) & wish & /hn'dzæt/ & حاجت \\
\hline hakikat (n) & reality & /hæqi:'qæt/ & حقيقت \\
\hline hakiki (adj) & real & /hæqi: 'qi:/ & حقيقى \\
\hline hakim (n) & judge & /hæ'ki:m/ & 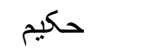 \\
\hline hal (n) & situation & $/ \mathrm{hpl} /$ & حال \\
\hline halal (adj) & lawful & /hæ'lpl/ & حلال \\
\hline hamil (adj) & pregnant & /hpme'le/ & حامله \\
\hline haram (adj) & forbidden & /hæ 'rom/ & 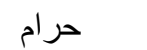 \\
\hline harmoni (n) & harmony & /hprməひ'ni:/ & هارمونى \\
\hline hasil (n) & product & /hp'sel/ & حاصل \\
\hline hasrat (n) & strong desire & /hæs'ræt/ & حسرت \\
\hline hawa (n) & the air & /hæ'vp/ & هوا \\
\hline hayat (n) & life & /hæ'jpt/ & حيات \\
\hline hidayat (n) & sign from God & /hedp'jæt/ & هدايت \\
\hline hijab (n) & veil & /he'dzpb/ & حجاب \\
\hline hijrah (n) & migration & /hedz'ræt/ & هجرت \\
\hline hikayat (n) & tale & /hekp'jæt/ & حكايت \\
\hline hikmat (n) & wisdom & /hek' mæt/ & حكمت \\
\hline hisab (n) & math & /he'spb/ & حساب \\
\hline hokum (n) & decree & /həठkm/ & 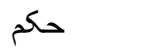 \\
\hline hotel (n) & hotel & /həช'tel/ & هتل \\
\hline hudud (n) & life limits & /həv'du:d/ & حدود \\
\hline huruf (n) & letter & /həu'ru:f/ & حروف \\
\hline ibadat (n) & worship & /ebp'dæt/ & عبادت \\
\hline Iblis (n) & Devil & /eb'li:s/ & ابليس ابل \\
\hline ihsan (n) & good deeds & /eh'spn/ & احسان \\
\hline ijazah (n) & permission & /edzp'ze/ & اجازه \\
\hline ikhtisar (n) & summary & /exte'spr/ & اختصار \\
\hline ikhtisas (n) & professional & /exte'sps/ & اختصاص \\
\hline iklim (n) & climate & /eq'li:m/ & اقليم \\
\hline iktiraf (v) & acknowledge & /e?te'rof/ & اعتر اف \\
\hline iktisad (n) & economy & /eqte'spd/ & افتصاد \\
\hline ilahi (adj) & divine & /elp'hi:/ & الهى \\
\hline ilham (n) & inspiration & /el'hpm/ & الهام \\
\hline ilmiah (adj) & academic & /el'miı/ & علميه \\
\hline ilmu (n) & knowledge & /elm/ & علم \\
\hline iltizam (n) & commitment & /elte'zom/ & التز ام \\
\hline imam (n) & imam & /e'mpm/ & امام \\
\hline iman (n) & faith in God & /i:'mon/ & ايمان \\
\hline imla (v) & dictate & /em'lp?/ & املاء \\
\hline inayat (n) & assistance & /enp'jæt/ & عنايت \\
\hline Inggeris (adj/n) & English & /engili'si/ & انكليسى \\
\hline ini (pron) & this & /i:n/ & اين \\
\hline Injil (n) & The Bible & /en'dzi:1/ & انجيل \\
\hline insan (n) & mankind & /en'spn/ & انسان \\
\hline institute (n) & institute & /ænsti'tu:/ & انستيتو \\
\hline istighfar (n) & prayers & /esteq'for/ & استغفار \\
\hline istilah (n) & terminology & /este'loh/ & اصطلاح \\
\hline isyak (n) & night time & /e'fp?/ & عثناء \\
\hline isyarat (n) & hint & /e $\int \mathrm{p}$ 're/ & اشتاره \\
\hline
\end{tabular}




\begin{tabular}{|c|c|c|c|}
\hline izin (n) & permission & /ezn/ & اذن \\
\hline jadual (n) & timetable & /dzæd'væl/ & جدول \\
\hline jahil (adj) & ignorant & /dzp'hel/ & جاهل \\
\hline jaket (n) & jacket & /30'kæt/ & زاكت \\
\hline jana (n) & life & /dzpn/ & جان \\
\hline janin (n) & fetus & /dzæ'ni:n/ & جنين \\
\hline jasad (n) & physical body & /dzæ'sæd/ & جسد \\
\hline jasmani (adj) & of the body & /dzes'moni:/ & جسمانى \\
\hline jasmaniah (adj) & of the body & /dzes'mpni:/ & جسمانى \\
\hline jawapan (n) & answer & /dzæ'vpb/ & جواب \\
\hline jenayah (n) & crime & /dzenv'jæt/ & جنايت \\
\hline jenazah (n) & corpse & /dzenn'ze/ & جنازه \\
\hline jenis (n) & nature & /dzens/ & جنس \\
\hline jidar (n) & edge & /dze'dpr/ & جدار \\
\hline jihad (n) & great effort & /dzæ'hpd/ & جهاد \\
\hline jilid (n) & book cover & /dzeld/ & جلد \\
\hline jin (n) & genie & /dzen/ & جن \\
\hline jisim (n) & body & /dzesm/ & جسم \\
\hline Jumaat (n) & Friday & /dzəơm' ' Pe/ & جمعه \\
\hline jumlah (n) & total & /dzəom'le/ & جمله \\
\hline kabel (n) & cable & $/ \mathrm{kpbl} /$ & كابل \\
\hline kabilah (n) & tribe & /qæbi: 'le/ & قبيله \\
\hline kabinet (n) & cupboard & /kpbi:'net/ & كابينت \\
\hline $\operatorname{kad}(\mathrm{n})$ & card & /kprt/ & كارت \\
\hline kadim (adj) & eternal & /qæ'di:m/ & قديم \\
\hline kafir (n) & infidel & /kp'fer/ & كافر \\
\hline kalam (n) & speech & $/ \mathrm{k} \mathfrak{x}^{\prime} \mathrm{lnm} /$ & كلام \\
\hline kanun (n) & set of laws & /qp'nu:n/ & قانون \\
\hline karavan (n) & caravan & /kpre'von/ & كاروان \\
\hline karib (adj) & close & /qæ'ri:b/ & قريب \\
\hline karikatur (n) & caricature & /kpri:kp'təur/ & كاريكاتور \\
\hline kartun (n) & carton & /kpr'təon/ & كارتن \\
\hline katib (n) & writer & /kp'teb/ & كاتب \\
\hline kebab (n) & kebab & /kæ'bpb/ & كباب \\
\hline $\operatorname{kek}(\mathrm{n})$ & cake & /keIk/ & كيى \\
\hline kelas (n) & class & /ke'los/ & كلاس - \\
\hline kerusi (n) & chair & /kəur'si:/ & كرسى \\
\hline khabar (n) & news & /xæ'bær/ & خبر \\
\hline khadam (n) & servant & /xæ'dæm/ & خدم \\
\hline khaki (adj) & brownish yellow & /xp'ki:/ & خاكى \\
\hline khalayak (n) & creatures & /xælp'jeq/ & خلايق \\
\hline khalifah (n) & caliph & /xæli:'fe/ & خليفه \\
\hline khas (adj) & special & /xps/ & خاص خاص \\
\hline khasiat (n) & propertiy & /xp'si:æt/ & خاصيت \\
\hline khat (n) & calligraphy & /xat/ & خط \\
\hline khatam (n) & end & /xp'tæm/ & خاتم \\
\hline khatib (n) & preacher & $/ \mathrm{x} \mathfrak{x}^{\prime} \mathrm{ti}: \mathrm{b} /$ & خطيب \\
\hline khatulistiwa (n) & equator & /'xætte əuste'vp/ & خط استو ا \\
\hline khayal (n) & dreaming & /xi:pl/ & خيال \\
\hline khianat (n) & betrayal & /xi:p'næt/ & خبانت \\
\hline khidmat (n) & service & /xed'mat/ & خدمت \\
\hline khilaf (n) & mistake & xæ'lpf/ & خلاف \\
\hline khunsa (n) & hermaphrodite & /хәชn'sp/ & خنتى \\
\hline
\end{tabular}




\begin{tabular}{|c|c|c|c|}
\hline khurafat (n) & superstitions & /xəorn'fpt/ & خر افات \\
\hline khusus (adj) & particular & /xəひ'su:s/ & خصوص \\
\hline khutbah (n) & sermon & /xəot'be/ & خطبه \\
\hline kiamat (n) & doomsday & /qi:p'mæt/ & 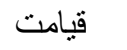 \\
\hline kimia (n) & chemistry & /ki:'mi:p/ & كيميا \\
\hline kimono (n) & kimono & /ki:məひ'nəひ/ & كيمونو \\
\hline kisah (n) & story & /qes'se/ & قصه \\
\hline kismis (n) & raisin & /ke $\int^{\prime}$ me $/$ & كشمش \\
\hline kitab (n) & holy book & /ke'tob/ & كتاب \\
\hline klinik (n) & clinic & /keli: 'ni:k/ & كلينيك \\
\hline kolej (n) & college & /kn'ledz/ & كالج \\
\hline korban (n) & sacrifice & /qəurbp'ni:/ & قربانى \\
\hline kot (n) & coat & /kəot/ & كت \\
\hline kubur (n) & grave & /qæbr/ & قبر \\
\hline kurma (n) & date & /xəur'mp/ & خرما \\
\hline lafaz (n) & pronunciation & /læfz/ & لفظ \\
\hline laknat (n) & damn & /læ?'næt/ & لعنت \\
\hline lampu (n) & lamp & /lpmp/ & لامب \\
\hline larva (n) & larva & /lprv/ & لارو \\
\hline lastik (n) & rubber & /los'ti:k/ & لاستيك \\
\hline lazim (adj) & common & /lp'zem/ & ل ازم \\
\hline $\operatorname{limau}(n)$ & lemon & /li:'mu:/ & ليمو \\
\hline loghat (n) & word & /ləひ'qæt/ & لغت ل ل \\
\hline loh $(\mathrm{n})$ & stone tablet & /lərh/ & لوح \\
\hline lokomotif (n) & locomotive & /ləukəuməひ'ti:v/ & وكومنيو \\
\hline madrasah (n) & school & /mædre'se/ & مدرسه \\
\hline maghrib (n) & dusk & /mæq'reb/ & مغرب \\
\hline mahir (adj) & expert & /mp'her/ & ماهر \\
\hline mahkamah (n) & law court & /mæhkæ'me/ & محكمه \\
\hline mahsul (n) & country's export & /mæh'su:1/ & محصول \\
\hline majalah (n) & magazine & /mædzæl'le/ & مجله \\
\hline majlis (n) & council & /mædz'les/ & مجلس \\
\hline majun (n) & traditional medicine & /mæ?dzu:n/ & معجون \\
\hline makalah (n) & article & /mæqp'le/ & 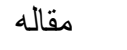 \\
\hline makam (n) & magnificent tomb & /mæ'qpm/ & مقام \\
\hline makaroni (n) & macaroni & /mpkprəo'ni:/ & ل اكارونى \\
\hline makbul (adj) & fulfilled & /mæq'bu:1/ & مقبول \\
\hline makhluk (n) & creature & /mæxlu:q/ & مخلوق \\
\hline makrefat (n) & knowledge & /mæ?re'fæt/ & معرفت \\
\hline makruh (n) & objectionable thing & /mæk'ru:h/ & مكروح \\
\hline maksiat (n) & $\sin$ & /mæ?'si:æt/ & معصيت \\
\hline maksud (n) & intention & /mæq'su:d/ & مقصود \\
\hline maktub (adj) & formally written & /mæk'tu:b/ & مكتوب \\
\hline manfaat (n) & benefit & /manfe' '?æt/ & منفعت \\
\hline mantik (n) & logic & /mæn'teq/ & منطق \\
\hline masalah (n) & problem & /mæs?æ'le/ & مسئله \\
\hline Masihi (n) & A.D. & /mæsi: 'hi:/ & مسيحى \\
\hline masjid (n) & mosque & /mæs'dzed/ & مسجد \\
\hline masyhur (adj) & famous & /mæ $\int^{\prime}$ hu:r/ & مشهور \\
\hline maujud (adj) & existent & /məv'dzu:d/ & موجود \\
\hline mazhab (n) & religious sect & /mæz'hæb/ & مذهب \\
\hline medal (n) & medal & /me'dpl/ & مدال \\
\hline menara(n) & tower & /menp're/ & مناره \\
\hline
\end{tabular}




\begin{tabular}{|c|c|c|c|}
\hline mesyuarat (n) & discussion & 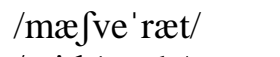 & مشورت \\
\hline mikrob (n) & microbe & /mi:k'rəob/ & ميكرب \\
\hline missal (n) & example & /me'spl/ & 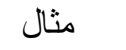 \\
\hline miskin (adj) & poor & /mes'ki:n/ & مسكين \\
\hline $\operatorname{model}(\mathrm{n})$ & model & /məv'del/ & مدل \\
\hline muafakat (n) & agreement & /məuvpfe 'qæt/ & مو افقت \\
\hline mualim (n) & teacher & /məv?æl'lem/ & معلم \\
\hline muazam (n) & majesty & /məひ२æz'zæm/ & معظم \\
\hline muazin (n) & prayer caller & /məひ?æz'zen/ & موذن \\
\hline muflis (adj) & bankrupt & /məof'les/ & مفلس \\
\hline muhrim (n) & family member & /mæh'ræm/ & محرم \\
\hline muhibah (n) & kindness & /mərheb'bæt/ & محبت \\
\hline mujarab (adj) & effective & /məudzær' ræb/ & مجرب \\
\hline mukjizat (n) & miracles & məvidze'zot/ & معجزات \\
\hline mukmin (n) & faithful in God & /mər?'men/ & مومن \\
\hline muktabar (n) & famous person & /məช?tæ'bær/ & معتبر \\
\hline munafik (adj) & hypocritical & /mərnn' feq/ & منافق \\
\hline munajat (v) & pray & /morno'dzpt/ & مناجات \\
\hline murid (n) & pupil & /məv'ri:d/ & مريد \\
\hline musabab (n) & cause & /məusæb'beb/ & مسبب \\
\hline musafir (n) & traveler & /məusp'fer/ & مسافر \\
\hline musim (n) & season & /mu:'sem/ & موسم \\
\hline mustaid (adj) & ready & /məustæ' 'ed/ & مستعد \\
\hline mustajab (adj) & effective & /məustæ'dzpb/ & مستجاب \\
\hline musyawarah (n) & discussion & 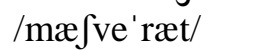 & مشورت \\
\hline musykil (adj) & difficult & /məð '́kel/ & مشكل \\
\hline musyrik (n) & infidel & /məひ' Jrek/ & مشرى \\
\hline muzakarah (n) & discussion & /məozpke're/ & مذاكره \\
\hline muzik (n) & music & /mu:'zi:k/ & موزيك \\
\hline nabi (n) & prophet & /næ'bi:/ & نبى \\
\hline nadir (n) & lowest point & /nv'der/ & نادر \\
\hline nafas (n) & breath & /næ'fæs/ & نفس \\
\hline nafi (v) & deny & /næfj/ & \\
\hline nafkah (n) & livelihood & /næfæ'qe/ & نفقه \\
\hline nafta (n) & oil & $/ \mathrm{næft} /$ & نفت \\
\hline nahas (adj) & unlucky & /næhs/ & نحس \\
\hline naib (n) & deputy & /np'jeb/ & نايب \\
\hline najam (n) & star & /nædzm/ & ن \\
\hline nakhoda (n) & captain & /nрхәひ'dp/ & ناخدا \\
\hline nama (n) & name & /npm/ & ن ام \\
\hline nasib (n) & chance & /næ'si:b/ & نصيب \\
\hline nasihat (n) & advice & /næsi: 'hæt/ & نصيحت \\
\hline nasihat (v) & advise & /næsi: 'hæt/ & نصيحت \\
\hline naskhah (n) & copy & /nəus'xe/ & نسخه \\
\hline Nasrani (n) & Christian & /næs'roni:/ & نصر انى \\
\hline nazam (n) & old poetry & /næzm/ & نظم \\
\hline nazir (n) & inspector & /nv'zer/ & ناظر \\
\hline niat (n) & intention & /ni:æt/ & نيت \\
\hline nikah (n) & marriage & /ne'kph/ & نكاح \\
\hline nisbah (n) & ratio & /ne'sbæt/ & نسبت \\
\hline nisbi (n) & relative & /nes'bi:/ & نسبي \\
\hline noktah (n) & full stop & /nəuq'te/ & نقطه \\
\hline $\operatorname{nur}(\mathrm{n})$ & light & /nu:r/ & نور \\
\hline
\end{tabular}




\begin{tabular}{|c|c|c|c|}
\hline nurani (adj) & bright & /nu:ro'ni:/ & او ران كَّ تانى \\
\hline orang utan (n) & orangutan & /u:ran gu:'ton/ & اور ان كونان \\
\hline orkid (n) & orchid & /əorki:'de/ & اركيده \\
\hline pahlawan (n) & warrior & /pæhlæ'vpn/ & يهلو ان \\
\hline pajama (n) & pyjamas & /pi:3p'me/ & بيزٍ امه \\
\hline papa (n) & father & $/ \mathrm{pp}$ 'pp/ & 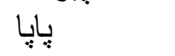 \\
\hline parket (n) & wooden floor & /ppr'ket/ & باركتُ \\
\hline Parsi (n) & Persian & /ppr'si:/ & يارسى \\
\hline parti (n) & party & /ppr'ti:/ & يارتى \\
\hline pasar (n) & market & /bp'zpr/ & باز \\
\hline penalty (n) & punishment & /penpl'ti:/ & ينالتى \\
\hline personel (n) & personnel & /persou'nel/ & ي يُرسنل \\
\hline piala (n) & cup & /pi:p'le/ & لِياله \\
\hline piknik (n) & picnic & /pi:k'ni:k/ & بِيك نِّى \\
\hline polis (n) & police & /pəo'li:s/ & يُ بِيس \\
\hline poster (n) & poster & /pəus'ter/ & يوستر \\
\hline projek (n) & project & /prəo'ze/ & 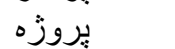 \\
\hline ragam (n) & type & /ræ' qæm/ & رقم \\
\hline rahib (n) & monk & /ro'heb/ & راهب \\
\hline rahim (n) & merciful & /ræ'hi:m/ & رحيم \\
\hline rahmat (n) & blessing & /ræh'mæt/ & رحمت \\
\hline rakyat (n) & people & /ræ?i:j'æt/ & رعيت \\
\hline resam (n) & custom & /ræsm/ & رسم \\
\hline rasmi (adj) & official & /ræs'mi:/ & رسمى \\
\hline rasuah (n) & bribe & /re $\int^{\prime} v e /$ & رشوه \\
\hline $\operatorname{rasul}(\mathrm{n})$ & messenger & /ræ'su:1/ & رسول \\
\hline rejam (v) & throw & /rædzm/ & رجم \\
\hline rejim (n) & regime & /re'zi:m/ & رزيم \\
\hline restoran (n) & restaurant & /restu:'ron/ & رستوران \\
\hline riadah (n) & hard exercise & /ri:p'zæt/ & رياضت \\
\hline rial (n) & currency & /ri:pl/ & " ربال \\
\hline ritma (n) & rhythm & /ri:tm/ & ريتم \\
\hline riwayat (n) & historical stories & /revp'jæt/ & روايت \\
\hline rohani (adj) & spiritual & /rəơh'ni:/ & روحانى \\
\hline rubah (n) & fox & /ru: 'bph/ & روباه \\
\hline sabun (n) & soap & /sp'bu:n/ & صابون \\
\hline $\operatorname{saf}(n)$ & line & /sæf/ & صف \\
\hline safari (n) & safari & /sæ'fær/ & سفر \\
\hline saham (n) & share & /sæhm/ & سهم \\
\hline sahih (adj) & true & /sæ'hih/ & صحيح \\
\hline saiz (n) & size & /saiz/ & سايز \\
\hline salasilah (n) & ancestry & /selse'le/ & سلسله \\
\hline salib (n) & crucifix & /sæ'li:b/ & صليب \\
\hline salih (adj) & pious & /sp'leh/ & صالح \\
\hline sandal (n) & sandal & /sæn'dæl/ & صندل \\
\hline sandwich (n) & sandwich & /spnde'vi:tf/ & ساندويج \\
\hline sebab (n) & cause & /sæ'bæb/ & سبب \\
\hline sejadah (n) & praying mat & /sædzdzp'de/ & سجاده \\
\hline selamat (adj) & safe & /sælp'mæt/ & سلامت \\
\hline selewat (n) & prayer for Prophet & /sælæ'vpt/ & صلو ات \\
\hline seminar (n) & seminar & /semi:'npr/ & سمينار \\
\hline serum (n) & serum & /se'rəom/ & \\
\hline siasah (n) & politics & /si:p'sæt/ & سياست \\
\hline
\end{tabular}




\begin{tabular}{|c|c|c|c|}
\hline sifar (n) & zero & /sefr/ & صفر \\
\hline sifat (n) & attribute & /se'fæt/ & صفت \\
\hline signal (n) & signal & /si:g'nol/ & سيكنال \\
\hline sihat (adj) & healthy & /seh'hæt/ & صحت \\
\hline sihir (n) & sorcery & /sehr/ & سحر \\
\hline sijil (n) & certificate & /se'dzel/ & سجل \\
\hline silo (n) & silo & /si:'lu:/ & سيلو \\
\hline sistem (n) & system & /si:s'tem/ & سيستر \\
\hline standard (n) & standard & /eston'dprd/ & ستاندارد \\
\hline subuh (n) & morning & /səubh/ & صبح \\
\hline sufi (n) & mysticism & /su:' fi:/ & سوفى \\
\hline sultan (n) & ruler & /səol'ton/ & سلطان \\
\hline sultanah (n) & kingdom & /sæltæ'næt/ & سلطنت \\
\hline $\sup (n)$ & soup & $/ \mathrm{su}: \mathrm{p} /$ & سوبٍ \\
\hline syah (n) & king & $/ \mathrm{gph} /$ & شَاْة \\
\hline syahadat (n) & creed & 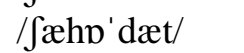 & شهادت \\
\hline syahid (n) & martyr & 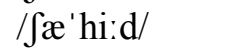 & شهيد \\
\hline syahwat (n) & lust & /Jæh'væt/ & شهوت \\
\hline syair (n) & poetry & $/ \int \mathrm{e} P \mathrm{r} /$ & شعر \\
\hline syal (n) & cloak & $/ \int \mathrm{pl} /$ & شال \\
\hline syampu (n) & shampoo & / $\mathrm{pm}$ 'pu:/ & شاميو \\
\hline syarat (n) & condition & / $æ r t / /$ & شرط ش \\
\hline syariat (n) & religious law & /Jæri: '?æt/ & شريعت \\
\hline syaarikat (n) & company & /Jer'kæt/ & شركت \\
\hline syeikh (n) & chieftain & $/ \int \mathrm{e}$ Ix/ & شيخ \\
\hline syukur (n) & gratefulness & 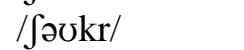 & شكر \\
\hline tabiat (n) & behavior & /tæbi:'?æt/ & طبيعت \\
\hline tabib (n) & physician & $/ \mathfrak{t æ}^{\prime} \mathrm{bi}: \mathrm{b} /$ & طبيب \\
\hline tabii (adj) & natural & /tæbi:'?i:/ & طبيعى \\
\hline tabligh (n) & presentation & /tæb'li:q/ & تبليغ \\
\hline tablo (n) & tableau & /tpb'ləఠ/ & نابلو \\
\hline tadbir (n) & management & /tæd'bi:r/ & تدبير \\
\hline tafakur (n) & meditation & /tæfæk'kəur/ & تفكر \\
\hline tafsir (n) & interpretation & /tæf'si:r/ & تفسير \\
\hline tahayul (n) & imagination & /tæxæj'jəðl/ & تخيل \\
\hline tahniah (n) & congratulation & /tæh'ni:æt/ & تهنيت \\
\hline takhta (n) & throne & $/$ tæxt/ & تخت \\
\hline takrif (n) & definition & /tæ?'ri:f/ & تعريف \\
\hline takwa (n) & Godliness & /tæq'vp/ & تقوا \\
\hline takwim (n) & calendar & /tæq' vi:m/ & تقويم \\
\hline takziah (n) & condolence & /tæ?' ziə/ & تعزيه \\
\hline talak (n) & divorce & $/$ tæ'lpq/ & طلاق \\
\hline madun (n) & civilization & /tæmæd'dəon/ & 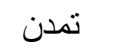 \\
\hline mat (adj) & finished & $/ \mathrm{tæ} \mathrm{mpm} /$ & تمام \\
\hline tamsil (n) & example & /tæm'si:1/ & ت تمثيل \\
\hline $\operatorname{tanur}(\mathrm{n})$ & oven & /tæn'u:r/ & تنور \\
\hline tasawuf (n) & Islamic mysticism & /tæsæv'vəof/ & تصوف \\
\hline tasbih (n) & praise to God & /tæs'bi:h/ & تسبيح \\
\hline ufan (n) & storm & /tu: 'fon/ & توفان \\
\hline ufik (n) & help from God & /təo'fi:q/ & نوفيق \\
\hline uhid (n) & belief in God & /təu'hi:d/ & نوحيد \\
\hline vakal (n) & reliance on God & /tævæk'kəひl/ & توكل \\
\hline nik (n) & technique & /tek'ni:k/ & تكنيك \\
\hline
\end{tabular}




\begin{tabular}{|c|c|c|c|}
\hline telefon (n) & telephone & /tele'fəon/ & تلفن \\
\hline televisyen (n) & television & /televezi:'ju:n/ & تلويزيون \\
\hline teori $(\mathrm{n})$ & theory & /tePəo'ri:/ & ت تئورى \\
\hline terjemah (v) & translate & /tærdzəv'me/ & ترجمه \\
\hline tertib (n) & order & $/$ tær'ti:b/ & ترتيب \\
\hline tohmah (n) & slander & /təo' 'mæt/ & تهمت \\
\hline trafik (n) & traffic & /tern'fi:k/ & تر افيك \\
\hline ufuk (n) & horizon & /əv'fəuq/ & افق \\
\hline umat (n) & followers & /əum' mæt/ & امت \\
\hline umum (adj) & general & /Рə઼'mu:m/ & عموم \\
\hline $\operatorname{umur}(n)$ & age & /Pəomr/ & ع عر \\
\hline unsure (n) & element & /Rəơn'səor/ & عنصر \\
\hline ustaz (n) & religious teacher & /əus'tpd/ & استاد \\
\hline wadi (n) & rocky watercourse & /vp'di:/ & و ادى \\
\hline wafat (v) & pass away & /væ'fpt/ & وفات \\
\hline wahid (adj) & one & /vp'hed/ & و احد \\
\hline wajib (adj) & compulsory & /vp'dzeb/ & و اجب \\
\hline wakaf (n) & devotion & /væqf/ & وقف \\
\hline wakil (n) & agent & /væ'ki:1/ & وكيل \\
\hline waktu (n) & time & /væqt/ & وقت \\
\hline wali (n) & guardian & /vp'li:/ & 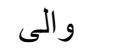 \\
\hline walimah (n) & feast & /væli:'me/ & وليمه \\
\hline waris (n) & heir & /vp'res/ & وارث \\
\hline wasiat (n) & will & /væ'si:æt/ & وصيت \\
\hline wasitah (n) & broker & /vose'te/ & واسطه \\
\hline waswas (n) & hesitation & /væs'vps/ & وسو اس \\
\hline wazir (n) & minister & /væ'zi:r/ & وزير \\
\hline wilayah (n) & province & /velp'jæt/ & ولايت \\
\hline wujud (v) & exist & /vəv'dzu:d/ & وجود \\
\hline Yahudi (n) & Jew & /jæhu:'di:/ & يهودى \\
\hline yakni (conj) & namely & /jæ'ni:/ & يعنى \\
\hline yatim (n) & orphan & /jæ'ti:m/ & يتيم \\
\hline zahid (adj) & ascetic & /zp'hed/ & زاهد \\
\hline zahir (adj) & external & /zp'her/ & ظاهر \\
\hline zaitun (n) & olive & /zei'tu:n/ & زيتون \\
\hline zalim (adj) & brutal & /zp'lem/ & ظالم \\
\hline zaman (n) & time & /zæ'mpn/ & زمان \\
\hline zarah (n) & particle & /zær're/ & ذره \\
\hline ziarah (n) & pilgrimage & /zi:p'ræt/ & زيارت \\
\hline zikir (n) & prayer & /zekr/ & ذكر \\
\hline zina (n) & adultery & /ze'np/ & زنا \\
\hline zirafah (n) & giraffe & /zærro'fe/ & زر افه \\
\hline zirah (n) & armor & /zær're/ & زره \\
\hline Zuhal (n) & Saturn & /zəひ'hæl/ & زحل \\
\hline Zuhrah (n) & Venus & /zəoh're/ & زهره \\
\hline zuhur (n) & noon & /zəohr/ & ظهر \\
\hline zuriat (n) & lineage & /zəor'ri:æt/ & ذريت \\
\hline
\end{tabular}




\section{APPENDIX B: Word Group 2}

Identical or very similar pronunciation, same meaning, and different part of speech

\begin{tabular}{|c|c|c|c|c|}
\hline Malay Word & $\begin{array}{l}\text { English Equivalent } \\
\text { for Malay Word }\end{array}$ & $\begin{array}{l}\text { Persian Equivalent } \\
\text { for Malay Word }\end{array}$ & $\begin{array}{l}\text { ian } \\
\text { Word } \\
\text { lunciation }\end{array}$ & $\begin{array}{l}\text { Persian } \\
\text { Key Word }\end{array}$ \\
\hline adab (adj) & polite & politeness (n) & ادب /æ'dæb/ & \\
\hline akibat (adj) & consequent & consequence (n) & /pqe'bæt/ & عاقبت \\
\hline baligh (n) & puberty & mature (adj) & /bo'leq/ بالغ & \\
\hline fana (adj) & ephemeral & inexistence (n) & /fæ'np/ فنا & \\
\hline hadir (v) & attend & present (adj) & حاضر /ho'zer/ حاضر & \\
\hline hafaz (v) & memorize & memorization (n) & /hefz/ حفظ & \\
\hline hasad (adj) & jealous & jeaulousy (n) & /hæ'sæd/ & حسد \\
\hline hormat (v) & respect & respect (n) & /həur' mæt/ & حرمت \\
\hline imla (v) & give orders giving $\mathrm{o}$ & orders $(n)$ & 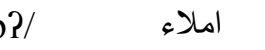 & \\
\hline imsak (v) & not drink or eat & $\mathrm{t} \quad$ fast $(\mathrm{n})$ & /em'spk/ امساك & \\
\hline ingkar (v) & deny & denial (n) & /en'kpr/ انكار & \\
\hline istirahat $(\mathrm{v})$ & rest & rest (n) & /estern'hæt/ & استر احت \\
\hline isytihar (v) & announce & reputation (n) & /efte'hor/ & اشتهار \\
\hline jawab (v) & answer & answer (n) & /dzæ'vpb/ & جواب \\
\hline keramat (adj) & holy & holiness (adj) & /kern' mæt/ & كر امت ك \\
\hline khalwat (v) & isolate oneself & isolation $(n)$ & /xæl'væt/ & خلوت \\
\hline lazat (adj) & joyful & enjoy (v) & /lez' zæt/لذت & \\
\hline lisan (adj) & oral & tounge & لسان /le'spn ل ل & \\
\hline maaf (n) & forgiveness & forgiven (adj) & /məひ'?pf/ & معاف \\
\hline majmuk (adj) & plural & $\operatorname{sum}(n)$ & /mædz'məひ?/ & مجموع \\
\hline mati (adj) & dead & death (n) & /məot/ موت & \\
\hline maut (adj) & lethal & death $(n)$ & /movt/ موت & \\
\hline muafakat (v) & agree & agreement (n) & /məuvpfe'qæt/ & مو افقت \\
\hline $\operatorname{murtad}(\mathrm{v})$ & convert religion & apostate $(\mathrm{n})$ & /məur'tæd/ & 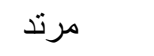 \\
\hline nujum (n) & astrologer & astrology (n) & /nəv'dzu:m/ & نجوم \\
\hline rahat $(\mathrm{v})$ & rest easy (adj) & /ro'hæt/ & راحت & \\
\hline rahmatullah (v) & pass away & passed away (adj) & /ræhmætəol'lph/ & رحمت اله/ \\
\hline rohani (n) & soul & spiritual (adj) & /rəohp'ni:/ & روحانى \\
\hline sabar (adj) & patient & patience (n) & /sæbr/ صبر & \\
\hline sahabat (n) & friend & friendship (n) & /məusphe'bæt/ & مصاحبت \\
\hline salam (v) & greet & greeting $(\mathrm{n})$ & سلام/sæ'lpm/ & \\
\hline sujud (v) & kneel down & kneel (n) & /səo'dzu:d/ & سجود \\
\hline syok (adj) & surprising & surprise (n) & 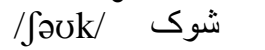 & \\
\hline taasub (adj) & prejudiced & prejudice (n) & /tæ?æs'səub/ & تعصب \\
\hline taat (adj) & loyal & loyalty (n) & طاعت /tb' 'جæt & \\
\hline warisan (n) & heritage & heirs (n) & /vpre'spn/ & و ارثنان \\
\hline yakin (adj) & certain & certainty (n) & /jæ 'qi:n/ يقين & \\
\hline
\end{tabular}




\section{APPENDIX C: Word Group 3}

Compound Malay words or expressions containing homophonic Persian segments

\begin{tabular}{|c|c|c|c|c|c|c|}
\hline $\begin{array}{l}\text { Malay } \\
\text { Word }\end{array}$ & $\begin{array}{l}\text { English } \\
\text { Equivalent for } \\
\text { Malay Word(s) }\end{array}$ & $\begin{array}{l}\text { English } \mathrm{E} \\
\text { for Persian }\end{array}$ & $\begin{array}{l}\text { Equivalent } \\
\text { in Key Word }\end{array}$ & $\begin{array}{l}\text { Key Word } \\
\text { Pronunciation }\end{array}$ & \multicolumn{2}{|l|}{ Key Word } \\
\hline ahli ma & & councilor & council (n) & /mædz'les/ & مجلس & \\
\hline ahli sih & & magician & magic (n) & /sehr/ & سحر & \\
\hline ahli suf & & a mystic & mystic (n) & /su: fi:/ & سوفى & \\
\hline akilbali & & puberty & wise (adj) & /p'qel/ & عاقل & \\
\hline askar u & $n(n)$ & mercenary & soldier (n) & /æs'gær/ & عسكر & \\
\hline bandar & (n) & capital city & port (n) & /bæn'dær/ & بندر & \\
\hline bandua & & prisoner & prison (n) & /bænd/ & بند & \\
\hline beradat & & customary & habit (n) & /p'dæt/ & عادت & \\
\hline berfikir & & think & thinking (n) & /fekr/ & فكر & \\
\hline berhijr & & migrate & migration (n) & /hedz'ræt/ & هجرت & \\
\hline berilmu & & knowledgeable & science (n) & /elm/ & علم & \\
\hline beristig & & repent & repent (n) & /esteq'fpr/ & استغفار & \\
\hline bersign & & signal & signal (n) & /si:g'npl/ & سيكنال & \\
\hline beriwa & & tell a story & story $(\mathrm{n})$ & /revp'jæt/ & روايت & \\
\hline berkala & $(\mathrm{adv})$ & periodically & merchandise (n) & $/ \mathrm{kp}$ 'lp/ & كالا & \\
\hline bermua & $\mathbf{t}(\mathrm{v})$ & discuss & agreement (n) & $/ m \partial u v p f e^{q}$ & مو افقت/æt| & \\
\hline bermus & $\operatorname{arah}(\mathrm{v})$ & discuss & discussion (n) & $/ m æ \int v e ' r æ t$ & مشورت / & \\
\hline bersala & & shake hands & greeting (n) & /sæ'lpm/ & سلام & \\
\hline bersara & & retire & feminine name & /sp'ro/ & سارا & \\
\hline bersyul & & be greatful & gratefulness (n) & / $\partial \partial u k r /$ & شكر & \\
\hline bertafa & & think deeply & thinking (n) & /tæfæk' kəひı & r/ تفكر r r & \\
\hline bertakz & & sympathize & sympathy (n) & /tæ?' zıə/ & تعزيه & \\
\hline bertaw & & rely on God & reliance (n) & /tævæk'kəu & توكل/1 & \\
\hline boleh d & hati (adj) & advisable & advice (n) & /næsi: 'hæt/ & نصبحت & \\
\hline bumipu & & natives of a cour & intry & local (adj) & /bu:'mi:/ & ل بومى / \\
\hline ekabah & & monolingual & discussion (n) & /bæhs/ & بحث & \\
\hline fikiran & & thought & thought & /fekr/ & فكر & \\
\hline huruf $b$ & & capital letter & letters (n) & /həひ'ru:f/ & حروف & \\
\hline $\begin{array}{l}\text { huruf r } \\
\text { ilmu al }\end{array}$ & & $\begin{array}{l}\text { Roman alphabet } \\
\text { geography }\end{array}$ & $\begin{array}{l}\text { Roman alphabet } \\
\text { science of world }\end{array}$ & $\begin{array}{l}\text { /həo'ru:f/+/ } \\
\text { /elm/ + /p'l }\end{array}$ & $\begin{array}{l}\text { lru:'mi:/ } \\
æ m /\end{array}$ & حروف + رومى علم عالم \\
\hline ilmuwa & & scholar & science $(\mathrm{n})$ & /elm/ & علم & \\
\hline ilmu ba & (n) & linguistics & science of langua & ages /elm/ + & + /bæhs/ & علم + بحث \\
\hline ilmu ba & & mysticism & science of soul & $/ \mathrm{elm} /+/ \mathrm{bp}^{\prime}$ & 'ten/ & علم + باطن \\
\hline ilmu ha & (n) & veterinary & science of animal & als $\quad /$ elm $/+$ & t/hei'von/ & علم + حيوان / \\
\hline ilmu hi & & mathematics & science of math & $/ \mathrm{elm} /+/ \mathrm{he}^{\prime}$ & spb/ ب & علم + حساب \\
\hline ilmu j & & psychology & science $(\mathrm{n})$ & /elm/ & علم & \\
\hline ilmu ke & an $(n)$ & medicine & science of health & / $/ \mathrm{elm} /$ + /seh & 'hæt/ & علم + صحت \\
\hline ilmu m & (n) & $\operatorname{logic}$ & science of logic & $/ \mathrm{elm} /+/ \mathrm{m} x$ & n'teq/ & علم + منطق \\
\hline ilmu sil & & sorcery & science of magic & /elm/ + /seh & & علم + سحر \\
\hline kefaha & & comprehension & comprehension & (n) $/ f æ h m /$ & | فهم | & \\
\hline kefana & & being transitory & inexistence (n) & $/ f æ n p /$ & فنا & \\
\hline kehan & & pregnancy & pregnant (adj) & /home'le/ & حامله & \\
\hline kehar & & illegality & illegal (adj) & /hæ' rom/ & حر ام & \\
\hline kelaza & & enjoyment & pleasure (n) & /lez'zæt/ & ل لذت & \\
\hline kemahi & & skill & skillful (adj) & /mp'her/ & ماهر & \\
\hline kenajis & & filthiness & filthy (adj) & /næ'dzes/ & نجس & \\
\hline
\end{tabular}




\begin{tabular}{|c|c|c|c|c|c|}
\hline kewujudan (n) & existence & existence (n) & /vəo'dzu:d/ د & وجود & \\
\hline lohmahfuz (n) & man's fate & fate $(n)$ & /ləohe mæh'fi & fu:z/ & لوح محفوظ \\
\hline majlis makan (n) & banquet & banquet (n) & /mædz'les/ c & مجلس & \\
\hline melazatkan (v) & make tasty & enjoy (v) & /lez'zæt/ & ل لذت & \\
\hline membahaskan (v) & argue & argument (n) & /bæhs/ & بحث & \\
\hline memisalkan (v) & liken & example (n) & /me'spl/ & 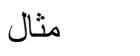 & \\
\hline memperadabkan (v) & civilize & politeness (n) & $/ \mathfrak{l}^{\prime} \mathrm{d} \mathrm{b} /$ & ادب اب & \\
\hline memungkari (v) & disobey & denier (n) & /məun'ker/ & منكر & \\
\hline menasihati $(\mathrm{v})$ & persuade & advice (n) & /næsi:'hæt/ & نصيحت & \\
\hline mendaftar (v) & register & notebook (n) & /dæf'tær/ & دفتر & \\
\hline mendandan (v) & adorn & tooth $(\mathrm{n})$ & /dæn'dpn/ & 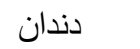 & \\
\hline mengabadikan (v) & immortalize & eternal (adj) & /æbæ'di:/ & ابدى ابد & \\
\hline mengamankan (v) & pacify & security (n) & /æ'mon/ & امان & \\
\hline mengamanatkan (v) & entrust & trust (n) & /æmb'næt/ & امانت & \\
\hline mengalamatkan $(\mathrm{v})$ & be a sign of & $\operatorname{sign}(n)$ & /ælp' mæt/ & علامت & \\
\hline mengalami $(\mathrm{v})$ & undergo & world (n) & /p'læm/ & 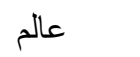 & \\
\hline mengizinkan (v) & permit & permit (n) & اذن /ezn/ اذ & & \\
\hline mengkhabarkan (v) & inform & news (n) & /xæ'bær/ & 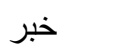 & \\
\hline mengkhianati (v) & betray & betrayal (n) & /xi:p'næt/ & خيانت & \\
\hline mengorbankan (v) & sacrifice & sacrifice (n) & /qəorbp'ni:/ & قربانى & \\
\hline menjahanamkan (v) & spoil & hell (n) & /dzæhæn'næn & جهنم & \\
\hline mentadbirkan (v) & mange & management (n) & /tæd'bi:r/ & ت ت تبير & \\
\hline mentafsir (v) & interpret & interpretation (n) & /tæf'si:r/ & تفسير & \\
\hline mentamsilkan (v) & illustrate & analogy $(\mathrm{n})$ & /tæm'si:1/ & تمثيل & \\
\hline meyakinkan (v) & assure & assurance (n) & /jæ'qi:n/ & يقين & \\
\hline mewajibkan (v) & compel & compulsory (adj) & $/ v p^{\prime} d z e b /$ & واجب & \\
\hline mewakilkan (v) & entrust & agent (n) & /væ'ki:1/ & وكين & \\
\hline mewariskan (v) & bequeath & heir (n) & /vp'res/ & و وارث & \\
\hline neraka jahanam (n) & hell & hell (n) & /dzæhæn'næn & جهنم & \\
\hline orang asli (n) & \multicolumn{5}{|c|}{ indigenous people original (adj) /æ'sli: / } \\
\hline orang jahil (n) & ignorant person & ignorant (adj) & /dzp'hel/ & 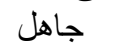 & \\
\hline Penasihat $(\mathrm{n})$ & advisor & advice $(\mathrm{n})$ & /næsi: 'hæt/ & نصيحت & \\
\hline penjenayah (n) & criminal & crime $(\mathrm{n})$ & /dzenv'jæt/ & جنايت & \\
\hline penjilid (n) & binder & book cover (n) & /dzeld/ & ج" جلد & \\
\hline perbandaran (n) & municipality & port (n) & /bæn'dær/ & 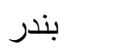 & \\
\hline peribumi (n) & aborigines & local (n) & /bu:'mi:/ & 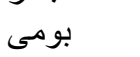 & \\
\hline perihal $(n)$ & condition & condition (n) & 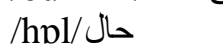 & & \\
\hline perikemanusiaan (n) & kindness & familiar (adj) & /mæ?'nu:s/ & مانوس & \\
\hline perkhidmatan (n) & service & service $(n)$ & /xed'mæt/ & خدمت & \\
\hline perkhidmatan awam (n) & civil service & service (n) & /xed'mæt/ & خ خدت & \\
\hline perkhidmatan sisial (n) & social service & service (n) & /xed'mæt/ & خدمت & \\
\hline perkuburan (n) & cemetery & graves (n) & /qәб'bu:r/ & قبور ق & \\
\hline permaafan (n) & forgiveness & exempted (adj) & /məठ'?pf/ & معاف & \\
\hline permuafakatan (n) & contract & agreement (n) & /məひPpfe'qæt & et/ & مو افقت \\
\hline pernafasan $(n)$ & breathing & breath $(\mathrm{n})$ & /næ'fæs/ & نفس & \\
\hline pernikahan (n) & wedding & wedding (n) & /ne'kph/ & نكاح & \\
\hline persamaan (n) & equation & $\operatorname{order}(n)$ & /sp'mpn/ & سامان & \\
\hline persefahaman (n) & understanding & understanding (n) & ) /fæhm/ ? & فهم & \\
\hline persembunyian (n) & cache & foundation (n) & /bəơn'jpn/ i & بنيان & \\
\hline perwakilan (n) & deputation & attorney $(\mathrm{n})$ & /væ'ki:1/ & و وكيل & \\
\hline perwujudan (n) & existence & existence (n) & /vəo'dzu:d/ د & وجود & \\
\hline pewarisan (n) & inheritance & heir (n) & /vo'res/ & و وارث & \\
\hline rumah anak-anak yatim & (n) & orphanage & orphan (n) & /jæ'ti:m/ & 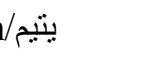 \\
\hline
\end{tabular}




\begin{tabular}{|c|c|c|c|c|c|}
\hline sangga (n) & stone & stone (n) & /sæyg/ & سنى سن & \\
\hline sezaman(adj) & contemporary & period (n) & /zæ'mpn/ & زمان & \\
\hline si bakhil (n) & miser & miser (n) & /bæ xi:1/ & بخيل & \\
\hline surat khabar (n) & newspaper & list + news & /su:'ræt. xæ'l & bær/ & صورت + خبر \\
\hline tamsilan (n) & analogy & analogy (n) & /tæm'si:1/ & تمثيل & \\
\hline tanggungjawab (n) & liability & answer (n) & /dzæ'vpb/ & جو اب & \\
\hline tataacara $(n)$ & agenda & work (n) & كار/kpr/ كار & & \\
\hline tataadat (n) & traditions & habit (n) & /Pp'dæt/ & 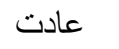 & \\
\hline tataakalimat (n) & syntax & words (n) & /kælæ' mpt/ & كلمات & \\
\hline tatatertib (n) & discipline & $\operatorname{order}(n)$ & /tær'ti:b/ & ترتيب & \\
\hline tengah hari (n) & midday & strait (n) & /tæy'ge/ & تنگه & \\
\hline terjamahan (n) & translation & translation (n) & /tærdzou' me/ & & ترجمه \\
\hline tida hadir (adj) & absent & present (adj) & /hp'zer/ & حاضر & \\
\hline tida sabar (adj) & impatient & patience (n) & /sæbr/ & صبر & \\
\hline Timur Tengah (n) & Middle East & strait (n) & /tæn'ge/ & تنحه & \\
\hline
\end{tabular}

\section{APPENDIX D: Word Group 4}

Identical or very similar pronunciation, and related shades of meaning

\begin{tabular}{|c|c|c|c|c|}
\hline Malay Word & $\begin{array}{l}\text { English Equivalent } \\
\text { for Malay Word }\end{array}$ & $\begin{array}{l}\text { Persian Equivalent } \\
\text { for Malay Word }\end{array}$ & $\begin{array}{l}\text { Persian } \\
\text { Key Word } \\
\text { Pronunciation }\end{array}$ & $\begin{array}{l}\text { Persian } \\
\text { Key Word }\end{array}$ \\
\hline abad (n) & century & eternity (n) & / ابد /æ'bæd & \\
\hline abah (n) & father & ancestors (n) & 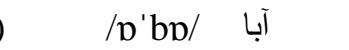 & \\
\hline aib (adj) & ashamed & fault $(n)$ & 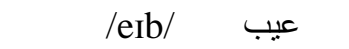 & \\
\hline akhbar (n) & newspaper & news (n) & /æx'bpr/ اخبار & \\
\hline alamat (n) & address & $\operatorname{sign}(n)$ & /ælp'mæt/ & علامت \\
\hline $\operatorname{arwah}(\mathrm{n})$ & the dead & ghosts (n) & ارواح/ær'vph & \\
\hline bahalul (adj) & not very clever & (fictional) nar & ame (n) /bəoh'lu:1/ & بهلول \\
\hline bahasa (n) & language & discussion $(\mathrm{n})$ & 1) بحث /bæhs/ & \\
\hline bandar (n) & town & port (n) & /bæn'dær/ & 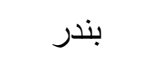 \\
\hline bumi (n) & earth & local (adj) & /bu:'mi:/ بومى & \\
\hline contoh (n) & example & like you & 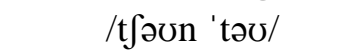 & جون تو \\
\hline daftar (n) & official list & notebook (n) & /dæf'tær/ & " دفتر \\
\hline dahsyat (adj) & awful & fear $(n)$ & /deh'Jæt/ & دهشت \\
\hline dakwa (v) & sue & fight (n) & /dæ?'vp// دعو/ & \\
\hline diraja (n) & royal & $\operatorname{rank}(\mathrm{n})$ & /dæræ'dze/ & درجه \\
\hline gourmet (n) & gourmet & fricassee (n) & /qəor' me/ & قرمه \\
\hline halwa (n) & fruit in sugar & a kind of swe & eet snack (n) /hæ'lvr & $\mathrm{vp} /$ \\
\hline hemat (adj) & sensible & effort (n) & /hem'mæt/ & همت \\
\hline ijazah (n) & diploma & permission $(\mathrm{n}$ & /edzp'ze/ & 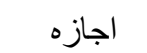 \\
\hline iradat (n) & God's will & will (n) & اراده /ern'de/ & \\
\hline jahanam (n) & wicked person & hell (n) & /dzæhæn'næm/ & جهiم \\
\hline jarrah (n) & plunder & sore (n) & جرح /dzærh/ & \\
\hline kabut (n) & fog & livid (adj) & $/ \mathrm{kæ}$ 'bu:d/ & كبود \\
\hline kalimah (n) & what was said & word $(n)$ & /kælæ'me/ & كلمه \\
\hline kalimat (n) & word & words (n) & $/ \mathrm{kælæ}^{\prime} \mathrm{mpt} /$ & كلمات \\
\hline labu (n) & gourd & beets $(n)$ & لبو /læ'bu:/ & \\
\hline lastik (n) & catapult & lastic (n) & لاستيك /lps'ti:k & \\
\hline mahdi (n) & a great leader & Messiah (n) & /mæh'di:/ & مهذى \\
\hline makluman (n) & notification & known (adj) & /mæ2'lu:m/ & معلوم \\
\hline
\end{tabular}




\begin{tabular}{|c|c|c|c|}
\hline maktab (n) & college & school (n) & /mæk'tæb/ \\
\hline mungkar (v) & defy God's laws & denier (n) & /məon'ker/ \\
\hline nikmat (adj) & enjoyable & blessing (n) & /ne?' mæt/ \\
\hline ranggi (adj) & handsome & colored (adj) & /ræy 'gi:/ رنكىى \\
\hline saat (n) & second & hour (n) & /sp'?æt/ ساعت \\
\hline sangkar (n) & cage & trench $(\mathrm{n})$ & /sæy'gær/ \\
\hline syarah (n) & lecture & explanation (n) & شرح شærh/ \\
\hline nah (n) & surface & body (n) & تنه /tæ'ne/ \\
\hline temasya (n) & show & spectacle (n) & $/$ tæmp' $\int \mathfrak{p} /$ \\
\hline
\end{tabular}

\section{APPENDIX E: Word Group 5}

Identical or very similar pronunciation, and different meaning

\begin{tabular}{|c|c|c|c|c|}
\hline Malay Word & $\begin{array}{l}\text { English Equivalent } \\
\text { for Malay Word }\end{array}$ & $\begin{array}{l}\text { ian Equivalent } \\
\text { Malay Word }\end{array}$ & $\begin{array}{l}\text { sian } \\
\text { Word } \\
\text { nunciation }\end{array}$ & $\begin{array}{l}\text { Persian } \\
\text { Key Word }\end{array}$ \\
\hline alan (n) & now (adv) & $/ \mathfrak{x}^{\prime} \operatorname{lnn} /$ & الان & \\
\hline amanat (n) & will & safekeeping (n) & /æmp'næt/ & امانت \\
\hline andam (v) & adorn & body $(\mathrm{n})$ & /æn'dpm/ & اندام \\
\hline $\operatorname{arus}(\mathrm{n})$ & flow & bride (n) & /æ'ru:s/ عروس & \\
\hline asak (v) & press & cane $(\mathrm{n})$ & $/ \mathfrak{x}^{\prime} \mathrm{sp} /$ & عصا \\
\hline awas (n) & caution & senses (n) & حواس /hæ'vds/ & \\
\hline ayam (n) & chicken & days (n) & /æj'jpm/ ايام & \\
\hline baghal (n) & mule & grocer (n) & /bæq'qpl/ & بقال \\
\hline bahana(n) & effect & excuse (n) & /bæho'ne/ & بهانه \\
\hline bahari (adj) & ancient & vernal (adj) & /bæhp'ri:/ & بهارى \\
\hline bani (n) & descendants & founder (n) & /bo'ni/ بانى / & \\
\hline baran (adj) & bad-tempered & rain $(n)$ & /bp'rnn/ بار ان & \\
\hline baru (adj) & new & fortification (n) & /bp'ru:/ بارو & \\
\hline bijan (n) & sesame & dead (adj) & /bi'dzpn/ & بيجان \\
\hline dahan (n) & bough & mouth (n) & /dæ'hpn/ دهان / & \\
\hline dandan (n) & hairstyle & tooth & /dæn'dpn/ & 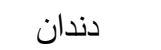 \\
\hline hari (n) & day & rabies $(\mathrm{n})$ & /hp'ri:/ هارى & \\
\hline hawar (n) & epidemic & loud shout & /hæ'vpr/ هوار & \\
\hline ibarat (n) & like & phrase (n) & /ebp'ræt/ & عبارت \\
\hline ikhtiar (v) & attempt & free will (n) & /ex'tipr/ اختيار & \\
\hline jahat (adj) & evil & direction (n) & /dzæ'hæt/ & جهت \\
\hline kala (n) & scorpion & merchandise (n) & كالا / & \\
\hline mahal (adj) & expensive & place $(n)$ & /mæ'hæl/ & محل \\
\hline makan (v) & eat & place (n) & /mæ'kpn/ & مكان \\
\hline mudarat (adj) & harmful & toleration (n) & /məudp'ro/ & مدارا \\
\hline munasabah (adj) & believable & occation (n) & /məunnse'bæt/ & مناسبت \\
\hline orang $(n)$ & people & throne (n) & /əu'ræng/ & اورنگ \\
\hline panah (n) & arrow & shelter (n) & / بناه/pæ'nph & \\
\hline raja $(n)$ & king & bliss (n) & رجا /جا /ræ'dzp & \\
\hline sakit (adj) & painful & silent (adj) & ساكت /sp'ket/ & \\
\hline salah (adj) & wrong & right (adj) & صلاح/sæ'lph/ & \\
\hline sangat (adv) & very & stone (n) & /sæyg/ سنگ & \\
\hline sara (n) & substance & feminine name & /sp'rp/ سارا & \\
\hline saraf (n) & nerve & money changer $(\mathrm{n}$ & صر اف/sær'rbf صر & \\
\hline sewa (n) & rent & separated $(\mathrm{adj})$ & سوا /sæ'vp/ & \\
\hline sukan (n) & sport & rudder $(\mathrm{n})$ & /səuk'kpn/ & سكان \\
\hline
\end{tabular}




$\begin{array}{ll}\text { surat }(\mathrm{n}) & \text { letter } \\ \text { surau (n) } & \text { praying room } \\ \text { tarik (v) } & \text { attract } \\ \text { tawan (v) } & \text { conquer } \\ \text { teriak (v) } & \text { cry } \\ \text { timur (n) } & \text { east } \\ \text { toleh (v) } & \text { look around }\end{array}$

\begin{tabular}{|c|c|}
\hline face $(n)$ & /su:'ræt/ صورت \\
\hline chapter (n) & /su:'re/ سوره / \\
\hline dark (adj) & تاريك /twr'i:k \\
\hline power (n) & /tæ 'vDn/ توان \\
\hline $\operatorname{ium}(n)$ & ترياك/ter'i:pk \\
\hline $\begin{array}{l}\text { lasculine name } \\
\mathrm{ub}(\mathrm{n})\end{array}$ & $\begin{array}{l}\text { /ter'mu:r/ } \\
\text { /tu:'le/ توله }\end{array}$ \\
\hline
\end{tabular}

